\title{
An Improved Perturbation and Observation Method for Power Transformer Active Noise Control
}

Q. Liu

HBEPC Technology Training Center (Wuhan Electric Power Technical College), Wuhan, China

J.W. Wang \& L.M. Ying

School of Electrical Engineering Wuhan University, Wuhan, China

X.C. Zhang \& L. Qi

HBEPC Technology Training Center (Wuhan Electric Power Technical College), Wuhan, China

ABSTRACT: There are lots of negative effects caused by transformer noise. The energy of the noise is mainly distributed in several low frequency points shown by analysis of transformer noise characteristics. In response to these characteristics, this paper proposed a new power transformer active noise control (ANC) system based on the perturbation and observation method. The method, designed simply and trackingeffectively, is employed in the system which can adjust the deviation of amplitude and phase quickly and reduce the energy of the noise in low frequency points. Simulation results confirm the effectiveness of the method.

KEYWORD: Perturbation and Observation Method; Transformer noise; Active Noise Control

\section{INTRODUCTION}

According to the sound environmental quality standard of China GB3096-2008, for urban residential area, the noise is not more than $55 \mathrm{~dB}$ during the daytime and $45 \mathrm{~dB}$ at night. By a certain substation site field measurement, it was found the substation station boundary noise is in $50 \pm 1 \mathrm{~dB}$, which close to the daytime noise standard limit value and above the nighttime standard limit value. This phenomenon also exists in the other similar location substations in this area. Due to the strong abilities of low frequency characteristics, penetrability and diffraction of power transformer noise ${ }^{1}$, the effect of traditional passive noise control methods, such as noise elimination, sound absorption and other ways, is not significant and the investment is huge. P. Leug proposed a concept of active noise control (ANC) in 1936, which provides a new solution to the problem of noise control ${ }^{2}$. To reduce sound energy and achieve noise reduction, ANC realize destructive interference in the sound field through sending a secondary sound wave which has the same amplitude and frequency, and $180^{\circ}$ phase difference as the noise in sound field, as shown in Figure 1. In view of the low frequency noise characteristics of power transformer, ANC is with smaller size, easy to design and control, easier to achieve phase compensation in the low frequency, and that it can achieve high precision in theory, when compared with the passive noise control.

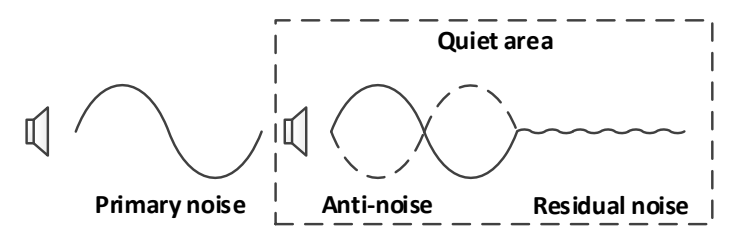

Figure.1 The principle of active noise control

By analyzing the characteristics of the transformer noise, this paper proposed an active noise control method based on disturbance observer, of which, $100 \mathrm{~Hz}, 200 \mathrm{~Hz}$, and $300 \mathrm{~Hz}$ frequency noises are selected as the target frequencies to be eliminated. The amplitudes and phases of the three target frequencies are automatically tracked and adjusted by the disturbance observer, respectively. And the controller outputs reverse waveform to eliminate noise. The simulation results show that the proposed method can effectively eliminate the noise. And through this study the foundation for the simulation experiment and engineering application of the active noise reduction system of power transformer is established.

\section{POWER TRANSFORMER NOISE ANALYSIS}

When active noise reduction method is used in different application environments of noise reduction, different control strategies have been adopted based on the differences of noise sources. Transformer noise is most itself main body noise, and it also includes auxiliary cooling device noise ${ }^{4}$. The main body noise is mainly caused by the vibration of iron core and tank, which induced by magnetostrictive 
effect. When the transformer core is as exciter, the size of silicon steel sheet shrinks at the direction perpendicular to the magnetic field lines, while the size increases at the direction along the magnetic field lines. Therefore, the core generates a periodic vibration according to the change of excitation frequency, the vibration frequency is twice as high as the power current frequency, then the vibration transfers through insulating oil and core pad passed and leads to tank vibration. So the transformer radiate noise to the outside world. And power system requires the power transformer to run stably for a long time. Hence, the transformer noise is not completely random uncertain noise, most noise energy is concentrated in the even times of power frequency, and its frequency spectrum is concentrated from $100 \mathrm{~Hz}$ to $600 \mathrm{~Hz}$. The change rate of baseband noise and 1 to 6 times harmonic noise is generally less than $0.4 \%$. And more than $80 \%$ of the noise energy is concentrated in the frequency from 100 to $600 \mathrm{~Hz}$ 5 . This study has collected a number of power transformer noise signals in multiple $110 \mathrm{kV}$ substations as the research object. One measurement data is selected to analysis in time domain and frequency spectrum. The time domain and the frequency spectrum of a measurement point is shown in Figure 2. At the same time, a transformer in the residential area is located at ten meters ahead of a measurement point, the sound pressure level of transformer noise is measured once every 10 minutes, the measurement results are shown in Figure 3.

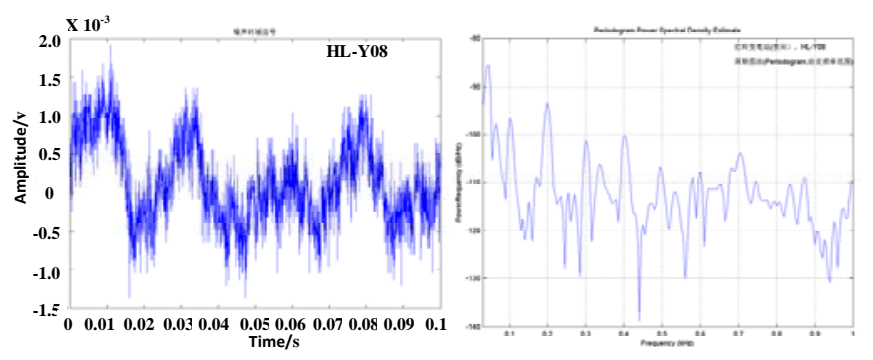

Fig.2 The time domain and frequency spectrum of one point in the substations

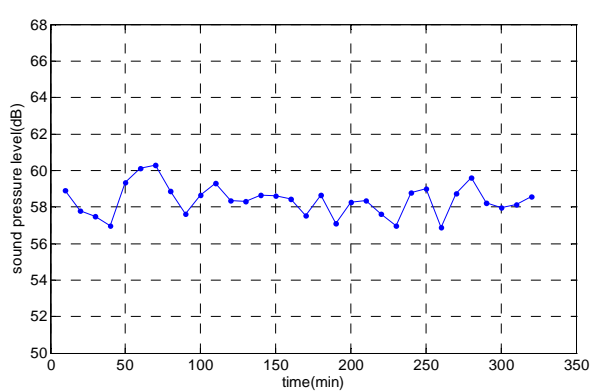

Fig.3 The sound pressure variation of the transformer noise

It can be seen from the measurement data and the results of spectrum analysis that the noise of transformer has a remarkable characteristic: 1) trans- former noise is stationary stochastic characteristic, and the variation is relatively smooth; 2 ) it has a discrete line spectrum characteristics; the energy distribution of discrete frequency points at $100 \mathrm{~Hz}, 200 \mathrm{~Hz}$, $300 \mathrm{~Hz}$ is larger, and the energy distribution of discrete frequency point at $100 \mathrm{~Hz}$ is the largest, and those at $200 \mathrm{~Hz}, 300 \mathrm{~Hz}, 400 \mathrm{~Hz}$ are decreased systematically. As shown in Figure 3, it can be seen that, in the continuous 320 minutes, the change rate of transformer sound pressure level (SPL) is relatively stable, small variation, and the noise SPL between the maximum and minimum value is no more than $3 \mathrm{~dB}$. In conclusion, it is known that the energy concentrated frequency component of transformer noise is fixed, the change rate of sound pressure is small, and acoustic environment is stable. In consideration of these specific noise features, an ideal noise reduction performance can be efficiently achieved by choosing appropriate control strategy to reduce the energy at frequency point of $100 \mathrm{~Hz}$, $200 \mathrm{~Hz}, 300 \mathrm{~Hz}$.

\section{DESCRIPTION OF THE ANC SYSTEM}

\subsection{Theory of Perturbation and Observation Method}

Perturbation and observation method is one of the most commonly used methods for maximum power point tracking in PV systems. Photovoltaic battery is expected to work at the maximum power output point $P_{\operatorname{MAX}}$ all the time, but with the change of sunshine and temperature in the environment, $P_{\mathrm{MAX}}$ changes dynamically. So the terminal voltage needs output voltage disturbance control repeatedly to guide the current reference voltage $U_{n}$ of the photovoltaic battery to change in a certain way according to the given voltage step change, and determine the power change at this time. If the $\Delta P>0$, change the voltage reversely; if the $\Delta P<0$, continue to change the voltage. By constantly monitoring the change of current output power of photovoltaic cells to determine the change direction of output voltage at next time, solar cells is made to work at the vicinity of the maximum power point, in order to realize the tracking of the maximum power output point $P_{\mathrm{MAX}}$. The principle is shown in Figure 4.

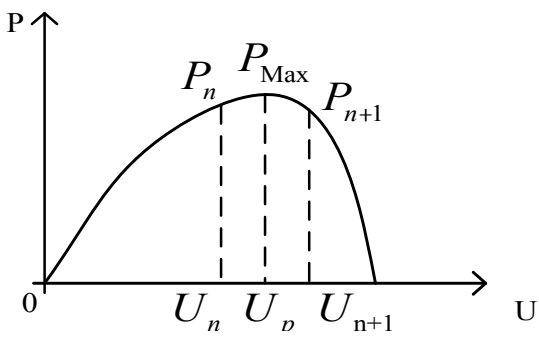

Figure.4 Basic principles of the perturbation and observantion method 


\subsection{ANC System Based on Improved Perturbation and Observation Method}

According to the analysis of second section, transformer noise signal is stable between two consecutive hours, the main energy is concentrated on $100 \mathrm{~Hz}, 200 \mathrm{~Hz}$ and $300 \mathrm{~Hz}$. In view of the low frequency noise characteristics of power transformer, the noise signal of $100 \mathrm{~Hz}, 200 \mathrm{~Hz}, 300 \mathrm{~Hz}$ which are extracted based on improved perturbation and observation method are given amplitude and phase perturbation. Adjust the size and direction of the perturbation according to the error margin of the before and after perturbation. Using the perturbation method can not only avoid the complex operation process, but also can quickly find the matching control signal, which has a good tracking performance.

The control flow of the ANC system based on the disturbance observation method is shown in Figure 5. The specific process is as follows:

1) The sensor signal of $100 \mathrm{~Hz}, 200 \mathrm{~Hz}, 300 \mathrm{~Hz}$ are extracted, and the amplitude and phase of each component are taken as the initial value of the control signal;

2) Using the disturbance observation module, firstly give amplitude and phase perturbation to the $100 \mathrm{~Hz}$ component, and then give perturbation and observation adjustment to the $200 \mathrm{~Hz}, 300 \mathrm{~Hz}$ component respectively;

3) Synthesizing the three frequency components.

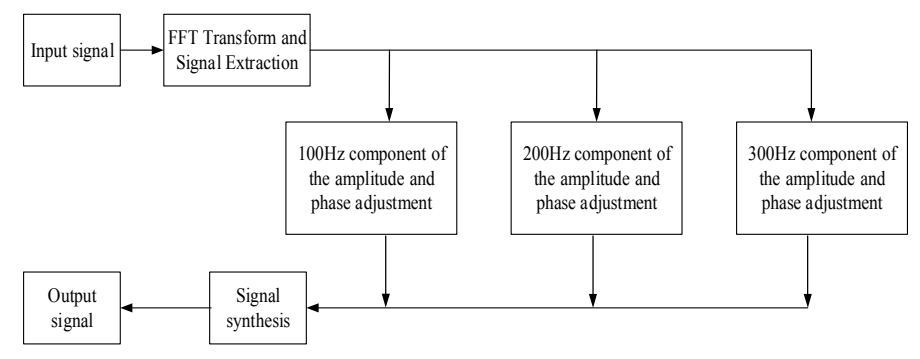

Figure.5 Control flow chart of ANC system

Amplitude and phase adjustment principle is shown in Figure 6. Amplitude and phase perturbations are carried out to the three frequency signal. Perturbations adjustment is determined by the error value so as to decrease it.

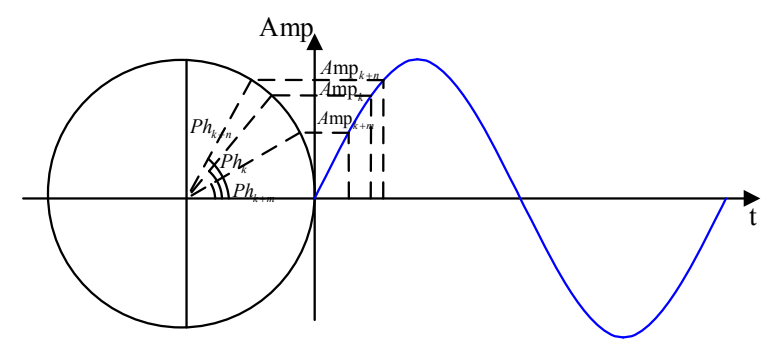

Figure.6 Basic principles of the perturbation and observantion method
The specific processes of the amplitude and phase adjustment module of which is shown in Figure 7. $\Delta_{k}$ is perturbation of amplitude or phase; $\lambda_{k}$ is step size of the perturbation; $k$ is number of perturbation; $\Delta(n)$ is reference of amplitude or phase; $e_{k}$ is error value; $\varepsilon$ is limit of the error value; $N_{\text {adj.max }}$ is limit of the number of perturbation.

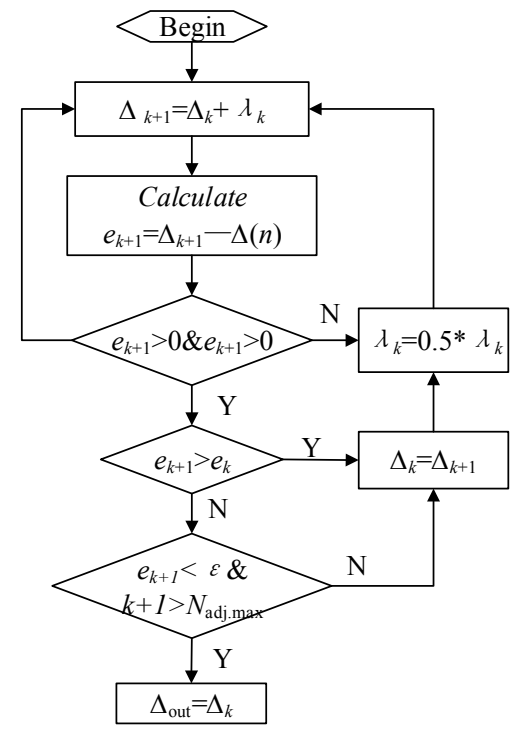

Figure.7 Flow chart of disturbance observation module

\section{SIMULATION RESULTS AND DISCUSSION}

In order to minimize the external interference, noise signal of the main transformer collected during the night in one $110 \mathrm{kV}$ substation are used as the primary noise signal in the simulation. In the experiment, the sampling frequency is $2.5 \times 10^{4} \mathrm{~Hz}$, the initial values of the ANC system based on the perturb \& observe algorithms are as follows: the initial phase and amplitude is 10 and 0.1 , the step of phase and amplitude are10 and 0.2, respectively, the maximum adjustment number is limited as 50 and the minimum error is limited as $1 \times 10^{-4}$. The noise component of $100 \mathrm{~Hz}, 200 \mathrm{~Hz}, 300 \mathrm{~Hz}$ which focus on the noise energy are the control target. Figure 7 represents the time domain of the primary noise and residual noise before noise reduction. The spectrum of the primary noise and residual noise are obtained by analyzing frequency characteristic of the noise using A weighting, which are shown in Figure 8 and Figure 9 , respectively. 


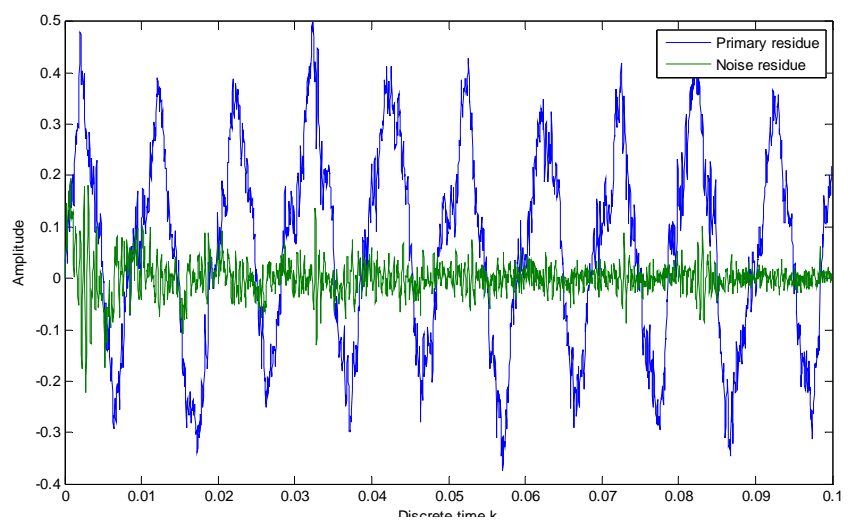

Figure. 8 Time domain of the primary noise and residual noise before noise reduction

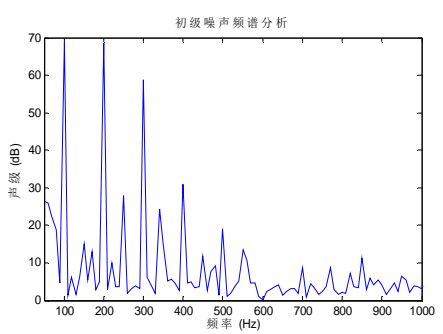

(a)

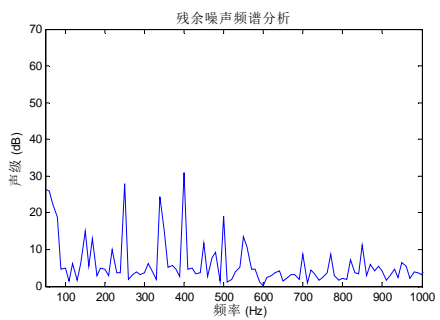

(b)
Figure.9 The spectrum of the primary noise

As mentioned in above works about noise characteristic of the transformer, we can know that $100 \mathrm{~Hz}$ is fundamental frequency and even multiple of $50 \mathrm{~Hz}$ is the harmonic frequency with discrete characteristic in line spectrum, and the frequency points of $100 \mathrm{~Hz}, 200 \mathrm{~Hz}, 300 \mathrm{~Hz}, 400 \mathrm{~Hz}$ have much more energy distribution. As seen from Figure 8, the energy distribution at $100 \mathrm{~Hz}$ is the largest, then followed by $200 \mathrm{~Hz}, 300 \mathrm{~Hz}, 400 \mathrm{~Hz}$. As shown in Figure 9, the noise energy at $100 \mathrm{~Hz}, 200 \mathrm{~Hz}, 300 \mathrm{~Hz}$ has been greatly suppressed, which is close to zero. Figure 7 depicts that effectively suppressing the noise of $100 \mathrm{~Hz}, 200 \mathrm{~Hz}, 300 \mathrm{~Hz}$ can offset the main energy of the primary noise and greatly reduce the residual noise. Thus, for controlling noise at $100 \mathrm{~Hz}, 200 \mathrm{~Hz}$, $300 \mathrm{~Hz}$, the active noise reduction system based on the perturb \& observe algorithms can quickly and effectively eliminate the main noise of the transformer. In addition, the system can be easily implemented with simple structure, and performances well at tracking the target frequency, responses fast and reduces the noise effectively with practical application value.

\section{CONCLUSION}

In this work, noise characteristics of the transformer are analyzed for active noise controlling the application environment. It is found that random characteristic of the noise signal is stable, the sound energy is concentrated at $100 \mathrm{~Hz}, 200 \mathrm{~Hz}, 300 \mathrm{~Hz}$ and the rate of sound pressure level has a small change. According to the noise characteristics of the transformer, by studying the thought of using the perturb \& observe algorithms as the adjustment strategy of active noise control system, adaptive active noise control method relying on the perturb \& observe algorithms is proposed and actual noise signals are collected to simulate. The simulation results show that the control system has a quick response and has a good behavior at tracking the target frequency, and can effectively eliminate the noise energy of the target frequency to achieve excellent noise reduction effect. Meanwhile, the system has advantages of simple implementation, controllable costs and precious value in further development of engineering application systems.

\section{REFERENCE}

C. Teoh, K. Soh, R. Zhou. 1998. Active Noise Control of Transformer Noise. Energy Management and Power Delivery 2: 747-753.

D.Y. Ma. 1987. Study of Noise Control. Beijing: Science Press 1: $232-302$.

D.P. Das, et al. 2013. Active Control of Transformer Noise by Using Power-Line Signal as Reference. 2013 IEEE 8th Conference on Industrial Electronics and Applications 1:953-956.

Enslin J, et al. 1997. Integrated Photovoltaic Maximum Power Point Tracking Converter. IEEE Trans. 1nd. Electron 44:769-773.

L.M. Zhang, et al, 2011. An algorithm for active control of transformer noise with a synthesized reference signal. Proceedings of the 40thInternational Congress \& Exhibition on Noise Control Engineering 1: 411-416.

P. Lueg. 1936. Process of Silencing Sound Oscillations. US Patent.

S. L. Foster \& E. Reiplinger. 1981. Characteristics and Control of Transformer Sound. IEEE Transactions on Power Apparatus and Systems 1(3): 131-141.

W.B. Conover. 1956. Fighting noise with noise. Noise Control 2: 78-82.

X.J. Qiu, C. H. Hansen. 2001. An algorithm for active control of transformer noise with on-line cancellation path modeling based on the perturbation method. Journal of Sound and Vibration 1(240): 647-665. 